\title{
Analytical Study of the Effect of Excessive Loading on Welding Residual Stress and Crack Growth near Piping Welds*
}

\author{
Jinya KATSUYAMA** and Kunio ONIZAWA** \\ ${ }^{* \star}$ Nuclear Safety Research Center, Japan Atomic Energy Agency \\ 2-4 Shirakata-Shirane, TOKAI-mura, NAKA-gun, IBARAKI, 319-1195, Japan \\ E-mail : katsuyama.jinya@jaea.go.jp
}

\begin{abstract}
Welding residual stress is one of the most important factors of stress corrosion cracking (SCC) growth in pressure boundary piping. The effect of excessive loading, such as an earthquake, on the residual stress is evaluated by axisymmetric thermo-elastic-plastic analyses based on the finite element method. After conducting welding residual stress simulation, several loading patterns of prescribed displacement for piping butt-welds have been applied in the axial direction by varying the maximum displacement and the combination of tension and compression. A greater displacement causes greater relaxation of welding residual stress near piping welds after the loading and unloading cycle. We conclude that the SCC growth rate could decrease as the amount of prescribed displacement increased.
\end{abstract}

Key words: Pressure Boundary Piping, Residual Stress, Stress Corrosion Cracking, Finite Element Method, Influence Function Method

\section{Introduction}

In the last decades, non-sensitized stress corrosion cracking (SCC) has been observed in the welds of core internal and pressure boundary piping in nuclear power plants. Residual stress is one of the most important factors to assess structural integrity related to an SCC event because high tensile stresses at the inner surface and through-thickness distribution in a pipe wall affect the initiation and growth rate of SCC. The Japanese seismic design guideline was revised in September 2006 and the Niigataken Chuetsu-oki Earthquake occurred in July 2007. Both of these events have focused attention on the need to assess the effect of large scale earthquake beyond the previous design base for the seismic structural integrity of reactor components. Therefore, it is necessary to take into account the effect of excessive loading on piping with SCC susceptibility for the structural integrity assessment after a large earthquake. In this study, several patterns of uniaxial loading for piping butt-welds after welding residual stress simulation were applied. We evaluate the effect of excessive loading on the redistribution of residual stress through the pipe thickness based on finite element method (FEM) analysis.

\section{Analytical method}

Thermo-elastic-plastic analyses of axisymmetric FEM models using ABAQUS (Ref. 1) have been conducted to evaluate the residual stress distribution near the butt-welding zone of $250 \mathrm{~A}$ pipes. The accuracy of the welding residual stress simulation used in the present work has been verified by comparing the results of experiments and three-dimensional FEM

${ }^{*}$ Received 15 Oct., 2008 (No. 08-0723) [DOI: 10.1299/jmmp.3.563] 
simulations (see Ref. 2). The residual stress distribution in the axisymmetric model agreed well with experimental measurements and analytical results in the steady region of the three-dimensional model with the same welding conditions and shapes of piping and groove. (Here the steady region means the constant welding condition portion, excluding the start and stop regions.) Therefore, the evaluation of residual stress by axisymmetric simulation is effective only in the steady region, which covers most of a weld.

The FEM model is shown in Fig. 1. The element types are shell elements with four nodal points (element ID is CAX4, element number $=942$, node number $=1,025$ ). The minimum size of elements which construct a crack tip is about $3.2 \mu \mathrm{m}^{2}$. The model includes a postulated SCC with a depth of $1 / 4$ of the pipe thickness. The position of the postulated SCC is based on the data from actual power plants obtained in Ref. 3. When the stress distribution without the crack is calculated, the Multi-Point Constraints (MPC) option is applied to the crack during welding. The material in this model is Type 316L low-carbon stainless steel. Each material property such as thermal conductivity, thermal expansion and stress-strain relationship is temperature dependent. The stress-strain relationship in the plastic region is defined using kinematic hardening based on a bilinear approximation. The heat source during welding is moved in the direction normal to the element plane in the two dimensional model. This welding simulation was carried out taking into account the adhesion of the weld metal by welding, i.e. elements of bead under each welding pass were added into the FEM model simultaneously with passage of the heat source. For this welding there are five layers and eight passes, as shown in Fig. 1. The shape of the heat source used is the so-called double ellipsoid model of Ref. 4 which simulates arc welding. The parameters of heat input and welding speed were derived from experimental results.

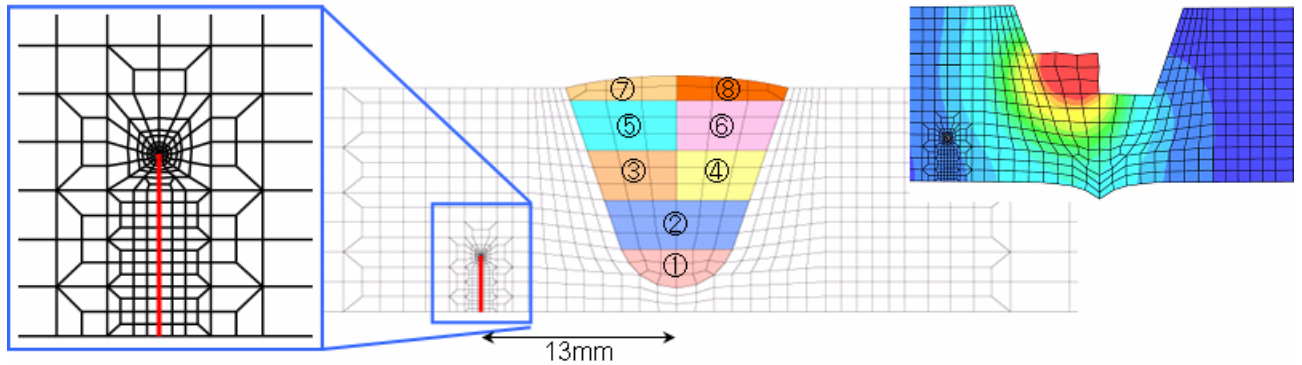

Fig. 1 Axisymmetric finite element model for welding residual stress simulation.

Excessive seismic loading was simulated by prescribing several uniaxial displacements with patterns which are parallel to the axial directions of piping butt-welds, as shown in Table 1. The prescribed displacement, from $0.01 \%$ to $1.0 \%$ in terms of strain, was applied to one end plane of the FEM model with the other end plane fixed. In Step 3 the prescribed displacement is unloaded by removing the constraints at the end plane. The redistribution of residual stress is then evaluated. The coefficients of the stress-strain relationship in the bilinear approximation, such as the Young's modulus, the yield strength and the work-hardening coefficient at room temperature are $199 \mathrm{GPa}, 310 \mathrm{MPa}$ and $40.2 \mathrm{MPa}$, respectively. Therefore, a prescribed displacement below about $0.16 \%$ in terms of strain corresponds to the elastic region.

SCC growth behavior was calculated under various residual stress distributions in

Table 1 - Prescribed displacement conditions

\begin{tabular}{|c|c|c|c|c|c|}
\hline & Step1 & Step2 & Step3 & $\begin{array}{l}\text { Loading displacement } \\
\text { (Step1/Step2) }\end{array}$ & Comments \\
\hline Case1 & Tension & - & \multirow{6}{*}{$\begin{array}{c}\text { Unloading } \\
\text { (Remove } \\
\text { constraints) }\end{array}$} & $0.01-1.0$ & \\
\hline Case2 & Compression & - & & $0.01-1.0$ & \\
\hline \multirow{2}{*}{ Case3 } & \multirow{2}{*}{ Compression } & \multirow{2}{*}{ Tension } & & $(0.01 / 0.01)-(1.0 / 1.0)$ & First and Second disp. is same \\
\hline & & & & $0.1 /(0.01-1.0)$ & First disp. is constant \\
\hline \multirow{2}{*}{ Case4 } & \multirow{2}{*}{ Tension } & \multirow{2}{*}{ Compression } & & $(0.01 / 0.01)-(1.0 / 1.0)$ & First and Second disp. is same \\
\hline & & & & $0.1 /(0.01-1.0)$ & First disp. is constant \\
\hline
\end{tabular}


piping with butt-welded joints based on a stress intensity factor (SIF) calculated by an influence function method for semi-elliptical cracks. Initial crack depth $(a)$ and initial crack length $(l)$ are defined as $0.1 \mathrm{~mm}$ and $3.18 \mathrm{~mm}$ respectively, corresponding to the median values of lognormal distributions in the NUREG report (Ref. 5). The crack is assumed to exist along a circumferential

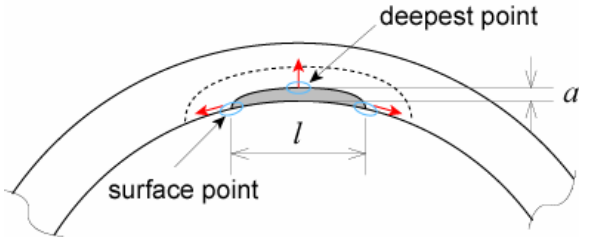

Fig. 2 Shape of crack and modeling parameters for crack. growth. direction, as shown in Fig. 2, and to propagate in the through-thickness direction. The SIF, or $\mathrm{K}$, is derived by the influence function method (Ref. 6) as follows.

$$
\begin{aligned}
& K=\left[A_{0} G_{0}+A_{1} G_{1}+A_{2} G_{2}+A_{3} G_{3}\right] \sqrt{\pi a / Q} \\
& \sigma=A_{0}+A_{1}(x / a)+A_{2}(x / a)^{2}+A_{3}(x / a)^{3} \\
& Q=1+4.593(a / l)^{1.62} \\
& (0 \leqq(a / l) \leqq 0.5,0 \leqq(a / t) \leqq 0.8)
\end{aligned}
$$

where, $a, l, x, t, \sigma$ and $G_{\mathrm{n}}$ are crack depth, crack length, distance from surface to crack tip (0 $\leqq(x / a) \leqq 1)$, pipe thickness, residual stress distribution and the coefficients of the influence function, respectively. SIF values are calculated using the through-thickness distribution of axial residual stress in a cross section where the postulated SCC exits. SCC growth behavior in low carbon stainless steel is evaluated by a crack growth rate diagram which shows crack growth rate as a function of SIF. In this work the diagram described in the JSME Rules on Fitness-for-Service for Nuclear Power Plants (Ref. 6) as shown in Fig. 3 is applied to the evaluation of crack growth. Crack growth rate is evaluated under a calculated welding residual stress distribution with axial membrane stress due to inner pressure by adding 98 $\mathrm{MPa}$ to $A_{0}$ in Eq. (2). This evaluation of crack growth is conservative because this axial stress due to internal pressure is equivalent to the design stress intensity of the stainless steel at the operating temperature.

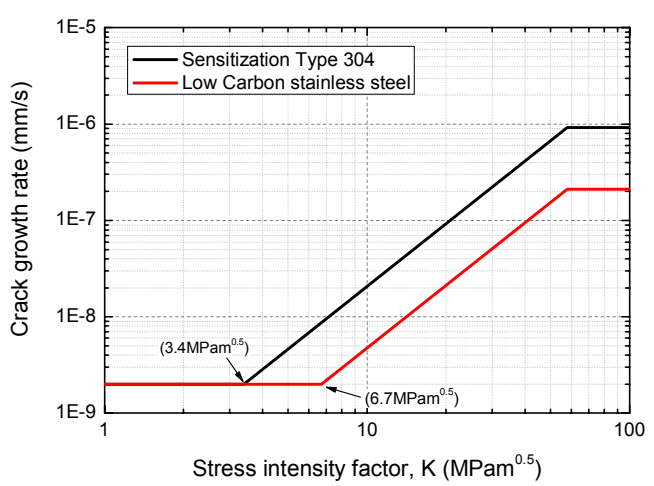

Fig. 3 Crack growth rate as a function of stress intensity factor provided by JSME code on FFS (Ref. 6).

\section{Welding Residual Stress Simulation}

Figure 4 shows axial and hoop residual stress distributions after welding. Through-thickness distributions of axial and hoop residual stresses at the cross section of the postulated SCC are also shown in Fig. 5. The axial residual stresses at the inner and the outer surfaces near the welding zone become tension and compression, respectively. Axial residual stress monotonically decreases as the through-thickness distance from the inner surface increases and it becomes compression near the outer surface. The hoop residual stress near the welding zone becomes tension in all regions, as shown in Figs. 4 and 5, 
although it gradually decreases by increasing through-thickness distance from the inner surface. These residual stress distributions have similar properties to those in Refs (7) and (8). The residual stress in the axial direction perpendicular to an SCC in the circumferential direction will be described in this paper, since SCCs in real components occur mainly along the hoop direction.
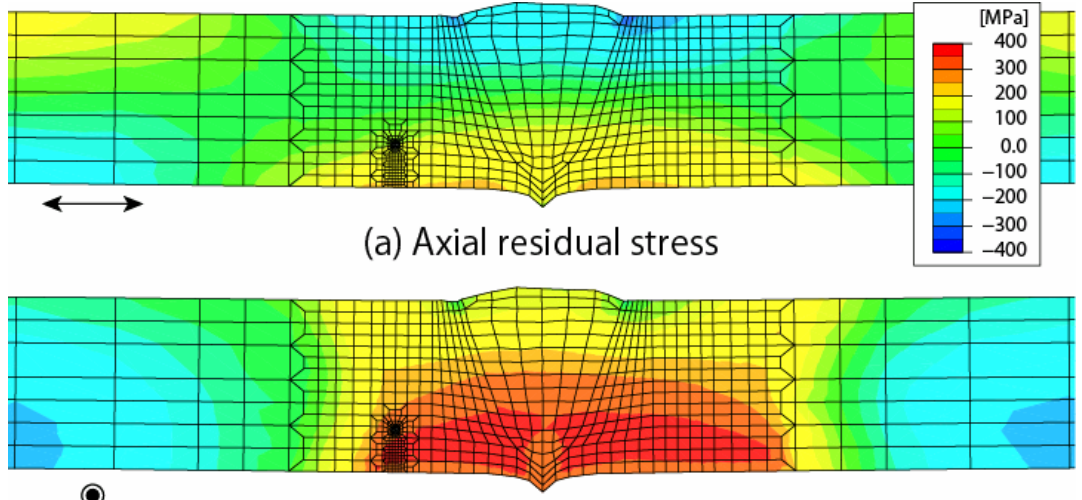

(b) Hoop residual stress

Fig. 4 (a) Axial residual stress and (b) hoop residual stress distributions after welding.

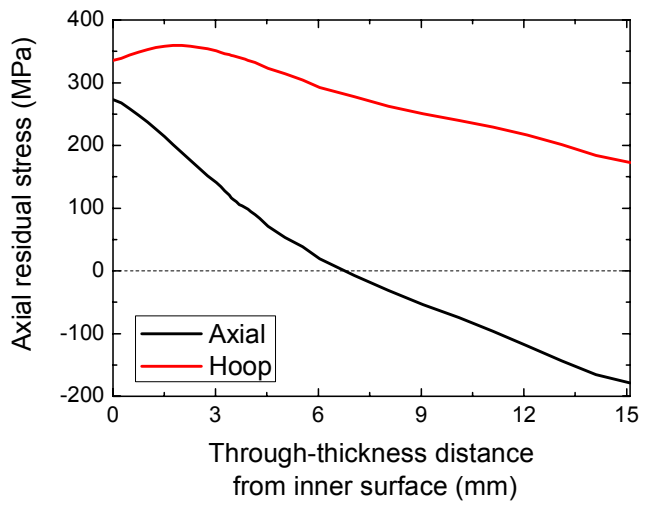

Fig. 5 Through-thickness distribution of axial and hoop residual stresses at the position of the postulated crack after welding.

\section{Excessive Loading Simulation}

Distributions of the axial residual stress along a cross section where a postulated crack exists after loading by the tension and compression displacements prescribed in Cases 1 and 2 are shown in Fig. 6. Closed and open symbols correspond to Case 1 and Case 2, respectively. Tensile residual stress at the inner surface decreases with the increase in displacement in both cases. In Case 1, residual stress at the inner surface decreases from $290 \mathrm{MPa}$ to $141 \mathrm{MPa}$ for a prescribed displacement of $0.1 \%$, which is in the elastic strain range. In Case 2 the effect of compression on the decrease of residual stress at the inner surface is larger than that of tension, e.g. residual stress decreases to $111 \mathrm{MPa}$ for a $0.1 \%$ displacement. Compressive residual stress at the outer surface also decreases in both cases as the prescribed displacement increases.

Figure 7 shows the change of through-thickness axial residual stress distributions as a result of loading by tension after compression (Case 3) and compression after tension (Case 4 ) at the same cross section shown in Fig. 6. Closed and open symbols correspond to Case 3 and Case 4, respectively. The magnitude of the second prescribed displacements (STEP 2) is 


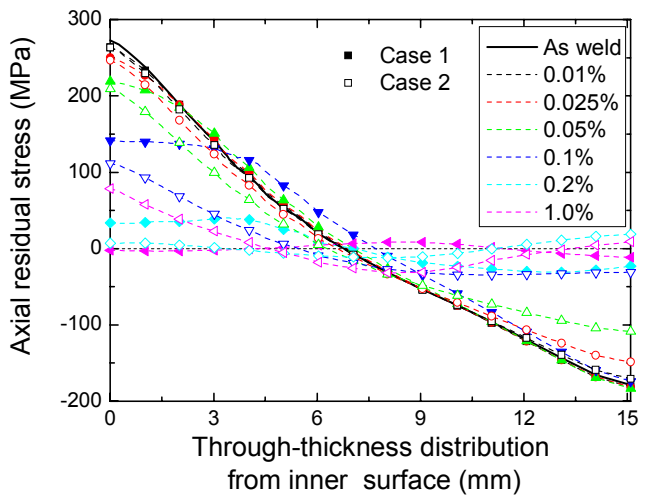

Fig. 6 Change of axial stress distribution for excessive loading at several levels (Case 1 and Case2).

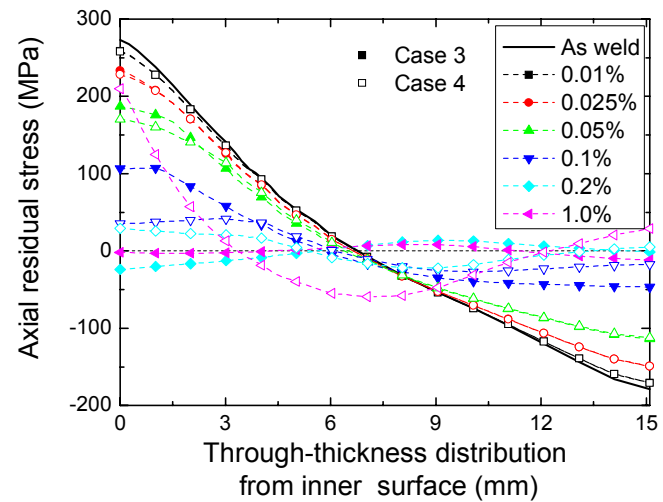

Fig. 7 Change of axial stress distribution for excessive loading at several levels (Case 3 and Case 4).

the same as the first (STEP 1). The behavior in these cases is similar to that of Case 1 and Case 2, i.e. higher excessive loading causes higher relaxation of welding residual stress near piping welds. The relaxation of residual stress by excessive loading is due to the redistribution of plastic strain near the welding zone. The plastic region of the welding zone is extended by excessive loading since the strain induces plasticity in adjacent parts of the welds. As a result, the residual stress from welding would decrease after excessive loading. However, the results of Cases 2 and 4 show that residual stress at the inner surface increases for the largest prescribed displacements of $1.0 \%$. The relaxation behavior of welding residual stress becomes complex in much larger excessive loading conditions such as large-scale yielding.

Figure 8 shows a comparison of the axial residual stress in the through-thickness direction for the same prescribed displacement of $0.1 \%$ in each case. Prescribed displacements of compression have larger influence on the redistribution of residual stress compared to tension, i.e. the decrease in tensile stress at the inner surface of Case 4 (Tens. + Comp.) is larger than that of Case 1 (Tens.). However, the residual stress of Case 3 (Comp. + Tens.) is almost the same as that of Case 2 (Comp.). The effects of excessive loading by tension and compression on the redistribution of residual stress are different.

To investigate this result in more detail, excessive loading simulations were performed in which the second prescribed displacement (STEP 2) in Case 3 and Case 4 was varied while the first prescribed displacement (STEP 1) is kept at a constant maximum value of $0.1 \%$ as shown in Fig. 9. The relaxation behaviors of axial residual stress at the inner surface of the cracked section are summarized in Fig. 10 for Cases 1 to 4 . Tensile residual stress at the inner surface drastically decreases after the first prescribed displacement of STEP 1 in each case. A decrease in residual stress can be observed after applying the second 
prescribed displacement in each case. However, some differences can be found after the loading of STEP 2 by comparing Case 3 with Case 4 in Figs. 9 and 10. The residual stress distribution does not change when the prescribed displacement is $0.1 \%$ in STEP 2 of Case 3. On the other hand, a redistribution of residual stress occurs for prescribed displacements below $0.1 \%$ in STEP 2 of Case 4. We speculate that such differences in the relaxation of residual stress by excessive loading arise from the fact that the source of welding residual stresses, such as tensile residual stress, is compressive plastic strain produced during welding. A detailed discussion on such a relationship between the relaxation of welding residual stress and the redistribution of plastic strain by excessive loading will be described in a separate paper.

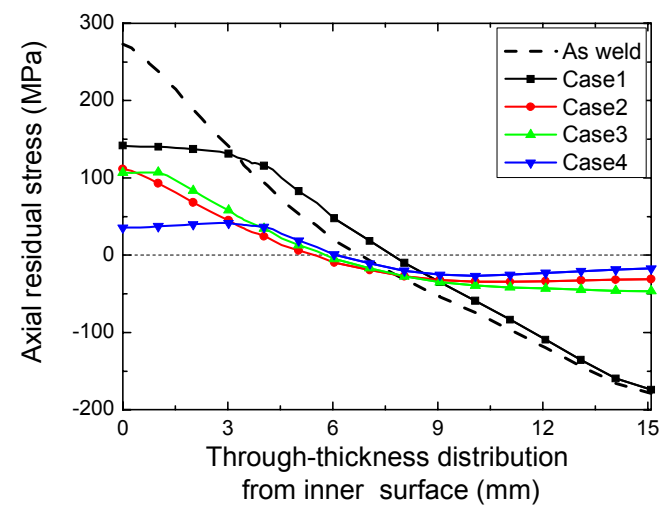

Fig. 8 Comparison of axial stress distribution for the same prescribed displacement of $0.1 \%$ in Cases 1 to 4 .

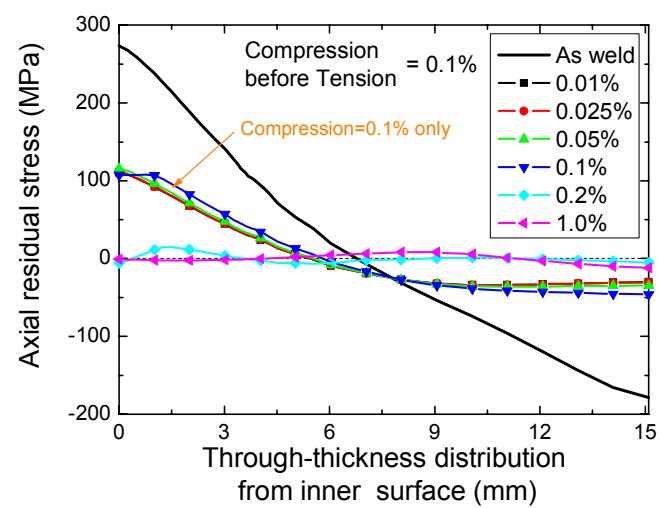

(a) Case 3

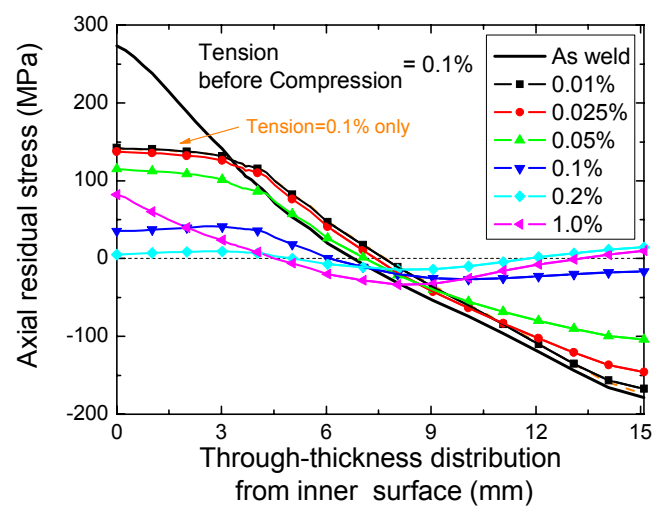

(b) Case 4

Fig. 9 Changes of through-thickness axial stress distribution by excessive loading at several levels for Case 3 and Case 4 (first prescribed displacement is constant at $0.1 \%$ ).

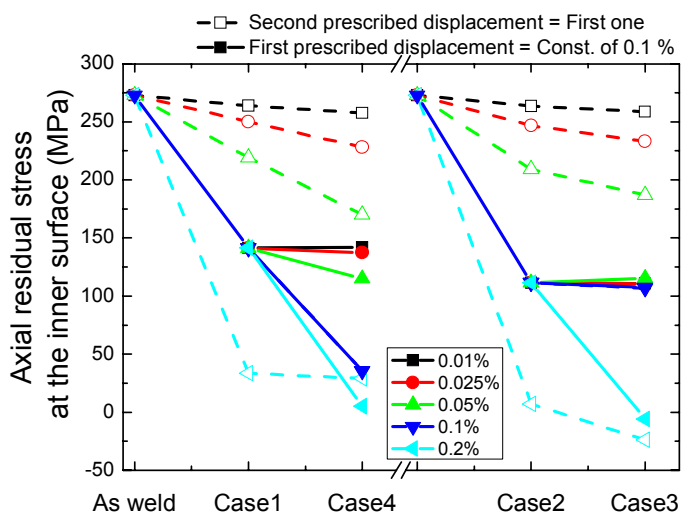

Fig. 10 Comparison of effect of excessive loading on the relaxation of axial residual stress at the inner surface for Cases 1 to 4 . 


\section{SCC Growth Simulation}

The crack growth analyses were performed by using residual stress distributions calculated by excessive loading simulation in cases where a postulated SCC exists. This analysis corresponds to the case that an earthquake occurs in early phase of operating, since the excessive loading simulation ignored the propagation of cracks. We note that a third-order polynomial curve fitting to Eq. (2) is a good approximation of the axial through-thickness stress distribution as shown in Figs. 6 to 10.

The SIF value at the deepest point of the crack tip and the crack depth are shown as a function of time in Fig. 11 for several loading levels in Case 3. The solid line with closed symbol shows the results for the as-welded condition. When the aspect ratio of crack becomes more than 0.5 , the SIF calculation was carried out by extrapolating the coefficients of $G_{\mathrm{n}}$ as indicated by the dotted line in this figure. The SIF value at the deepest point decreases as the prescribed displacement increases, since tensile residual stress near the inner surface decreases as the prescribed displacement is increased. As a result, the crack growth rate decreases with increasing prescribed displacement as shown in this figure. The time required for an SCC to propagate from $1 \mathrm{~mm}$ to $10 \mathrm{~mm}$ in depth, for a prescribed displacement of $0.1 \%$, is about twice as long as in the as-welded condition. Similar behaviors are found for the SIF value at the surface point of the crack and the crack length along the inner surface as shown in Fig. 12. The crack growth rate in the direction along the inner surface decreases with increasing prescribed displacement. The semi-elliptical crack tends to become a deep crack which has a large aspect ratio $(a / l)$ since the SIF at the deepest

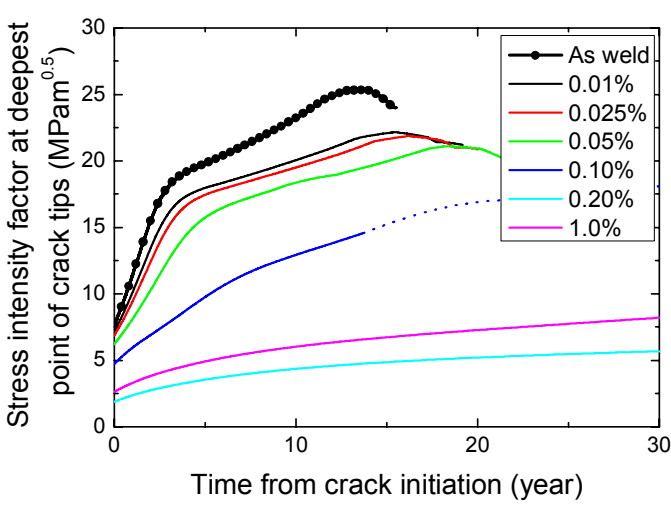

(a) Stress intensity factor

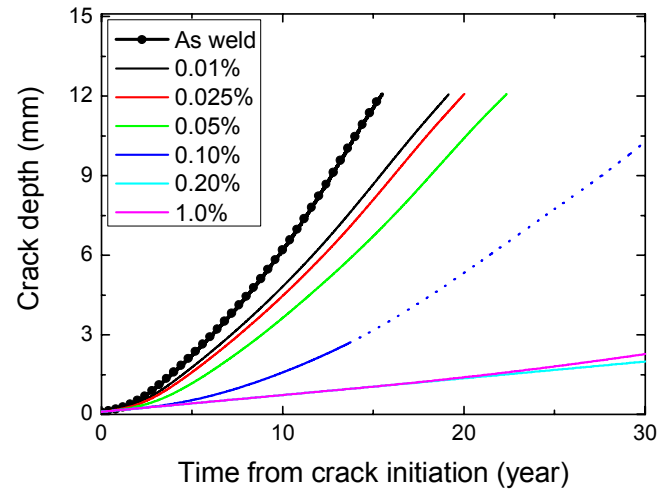

(b) Crack depth

Fig. 11 SCC growth behavior in the through-thickness direction under residual stress by welding and several levels of loading in Case 3.

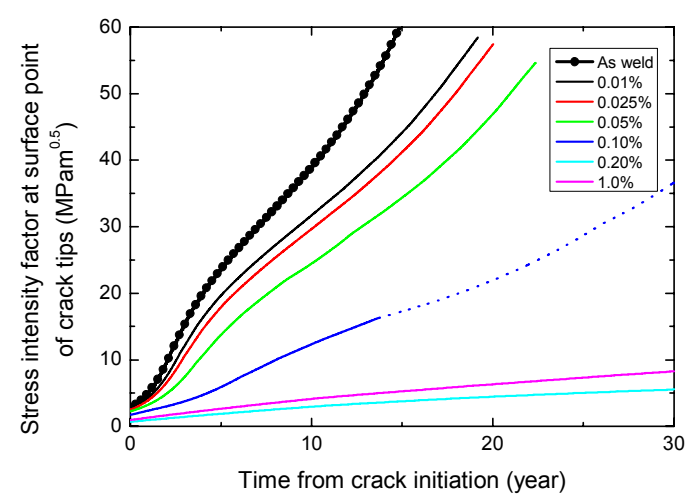

(a) Stress intensity factor

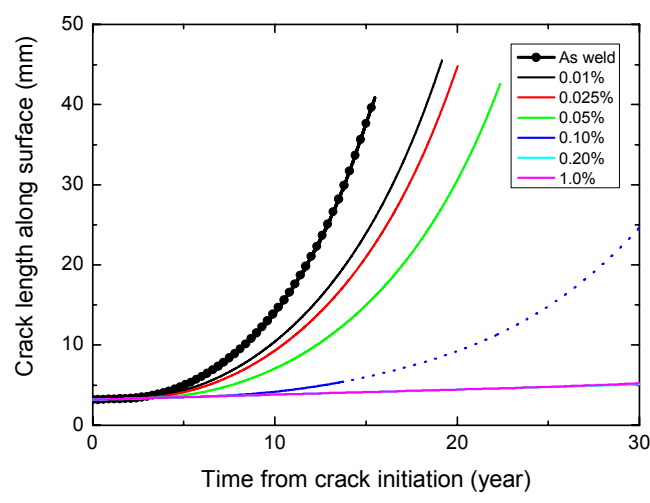

(b) Crack length

Fig. 12 SCC growth behavior along the inner surface under residual stress by welding and several levels of loading in Case 3. 


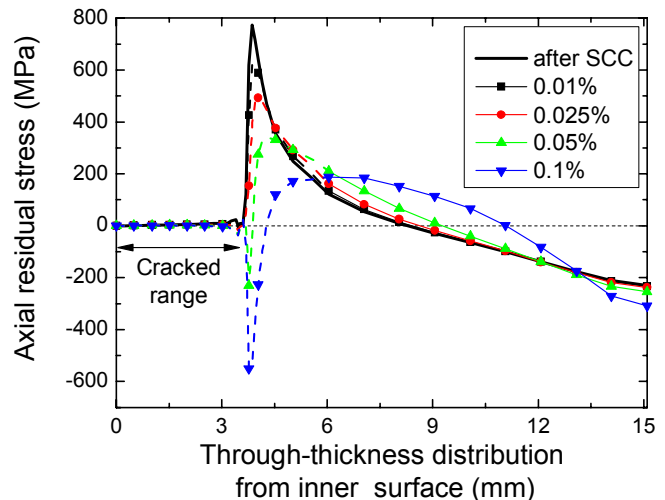

(a) residual stress

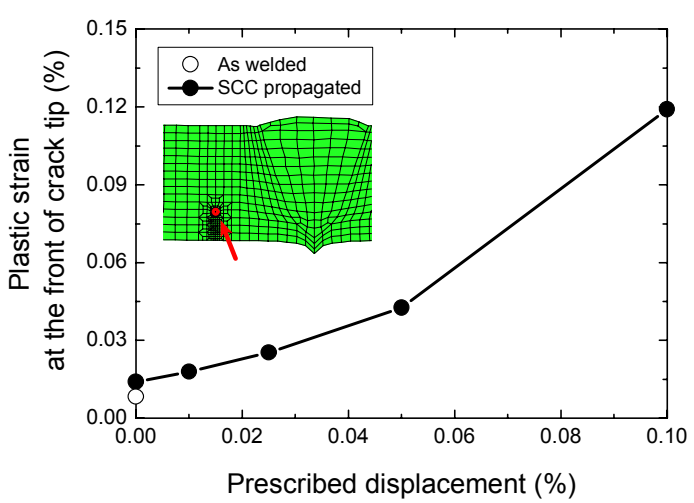

(b) plastic strain

Fig. 13 (a) Comparison of residual axial stress distributions by varying loading levels in Case 1 and (b) plastic strain in front of a crack tip as a function of prescribed displacement after the propagation of SCC.

point of the crack tip remains higher than that of surface points in the early growth stage. The time dependencies of SIF and crack growth in other cases have similar behaviors to those of Case 3 described above.

The effect of excessive loading on the redistribution of residual stress was evaluated in the case of a propagating SCC. The prescribed displacement is applied after the SCC has propagated to a depth of $3.8 \mathrm{~mm}$ (=1/4 of pipe thickness) by configuring the MPC option of the crack. The redistribution of residual stress for Case 1 is shown in Fig. 13. This figure also shows the change of plastic strain in front of the crack tip as a result of varying the prescribed displacement. High tensile stress exists in front of the crack tip as expected after the propagation of SCC. This stress decreases as the prescribed displacement increases. For prescribed displacements of $0.05 \%$ and $0.1 \%$, compressive stress occurs in front of the crack tip. Plastic strain in front of the crack tip increases as the prescribed displacement increases. Therefore, a suppressive effect on SCC growth is expected when an SCC is subjected to excessive loading. A similar suppressive effect of excessive loading on SCC growth based on experimental observations has been reported by Saito et al. (Ref. 9). This experimental effect may be explained as being the result of compressive plastic strain formation at the crack tip due to excessive loading.

\section{Summary}

The redistribution behavior of residual stress at butt-welding joints under excessive loading conditions simulating a large scale earthquake was evaluated by thermo-elastic-plastic analysis using an axisymmetric FEM model. The magnitude and direction of a prescribed displacement applied after welding simulation were varied. The analyses show that a greater displacement causes greater relaxation of welding residual stress near piping welds. Crack growth analyses were also performed using the calculated residual stress distribution. We conclude that excessive loading to piping butt-welds has an influence on the suppression of SCC growth due to the decreasing of tensile residual stress at the inner surface.

\section{References}

(1) ABAQUS Analysis User's Manual Version 6.7.

(2) J. Katsuyama, T. Tobita, H. Ito and K. Onizawa: "Effect of Welding Conditions on Residual Stress and SCC Behavior at Butt-Welding Joints of Recirculation Pipes," Proceedings of PVP 2008, PVP2008-61430 (2008). 
(3) Nuclear and Industrial Safety Agency (NISA), "On the Integrity Assessment Procedure of Primary Loop Recirculation Pipes," (in Japanese), (2004).

(4) J. Goldak, A. Chakravarti, and M. Bibby: "A new finite element model for welding heat sources," Metallurgical Transactions B, 15B (1984), p. 299.

(5) D. O. Harris, D. Dedhia and S. C. Lu, "Theoretical and User's Manual for pc-PRAISE, A Probabilistic Fracture Mechanics Computer Code for Piping Reliability Analysis," NUREG/CR-5864, UCRL-ID-109798 (1992).

(6) The Japan Society of Mechanical Engineers: Codes for Nuclear Power Generation Facilities - Rules on Fitness-for-Service for Nuclear Power Plants -, JSME S NA 1-2004 (2004), (in Japanese).

(7) M. Mochizuki, J. Katsuyama and M. Toyoda: "Effect of Geometric and Welding Conditions on Through-Thickness Residual Stress," Proceedings of PVP 2006, PVP2006-ICPVT11-93489 (2006).

(8) J. Katsuyama, W. Asano, K. Onizawa, M. Mochizuki and M. Toyoda: "Crack Growth Analyses of SCC under Various Residual Stress Distributions near the Piping Butt-Welding," Proceedings of PVP 2007, PVP2007-26574 (2007).

(9) T. Saito and M. Itow: "Retardation of SCC Propagation by Cyclic Overload," Proceedings of Materials \& Mechanics Conference 2008, GS0408 (2008), (in Japanese). 